\title{
- A Giant Nasal Septal Schwannoma - A Case Report
}

\section{Swain SK ${ }^{1}$, Kar Dattatreya ${ }^{2}$}

IJCRR
ection: Healthcare
ISI Impact Factor
(2019-20): 1.628
Value (2019): 90.81
SJIF (2020) = 7.893
Cc) 8)

\author{
'Department of Otorhinolaryngology, IMS and SUM Hospital, Siksha "O” Anusandhan University, K8, Kalinganagar, \\ Bhubaneswar-751003, Odisha, India; "Medical Research Laboratory, IMS and SUM Hospital, Siksha "O" Anusandhan University, K8, \\ Kalinganagar, Bhubaneswar-751003, Odisha, India.
}

\section{ABSTRACT}

Introduction: Schwannomas are benign neurogenic tumours which rarely found in the sinonasal area. However, the schwannoma at the nasal septum is extremely rare. A computed tomography (CT) scan confirmed the exact location of the tumour at the sinonasal tract. Endoscopic biopsy under local anaesthesia confirms the diagnosis of schwannoma.

Case Report: We report a case of a 32-year-old man who presented with left nasal obstruction for two months. Anterior rhinoscopy showed a smooth, firm and non-tender mass in the left nasal cavity arising at the posterior end of the nasal septum.

Result: CT scan of the paranasal sinus showed a mass at the posterior end of the nasal septum on the left side. The histopathological examination and immunohistochemical examination confirmed the diagnosis of the schwannoma.

Conclusion: Schwannomas are benign neoplasm and have an excellent prognosis with low potential for malignant transformation. It should be considered as the differential diagnosis of any mass arising from the nasal septum with a smooth surface. Surgical excision of the tumour is the primary treatment of choice in the case of nasal septal schwannoma. The transnasal endoscopic approach is considered the most commonly used approach for nasal septal schwannoma.

Key Words: Nasal septum, Schwannoma, Benign tumour, Trans-nasal endoscopic surgery, CT scan, Sinonasal tract

\section{INTRODUCTION}

Schwannomas are uncommon and benign neoplasm which originates from any peripheral, cranial or autonomic nerves which contain Schwann cells. ${ }^{1}$ These neoplasms are extremely rare in the nasal cavity. These tumours include schwannoma (neurilemmoma or neurinomas), neurofibromas and traumatic neuroma. Schwannoma is a neurogenic neoplasm arising from the sheath of myelinated nerve fibres. Majority of the head and neck schwannomas present as slowly enlarging, solitary, non-tender and encapsulated mass. Schwannomas are benign and encapsulated tumour which arises from the spinal roots, cervical nerves, sympathetic nerves, vagus, peroneal and ulnar nerves. Approximately $4 \%$ of the cases of schwannoma are found in the nasal cavity and paranasal sinuses. ${ }^{2}$ The nasal septum is considered the rarest site for the origin of the schwannoma. The current treatment approach is endonasal endoscopic resection of the tumour. ${ }^{3}$ The recurrence of this tumour is very rare and malignant transformation is also rare. ${ }^{3}$ Although there are very few cases of nasal septal schwannomas are reported in the medical literature, clinicians should think of this clinical entity in case of the mass found in the nasal septum. Here, we present a case of a large nasal septal schwannoma in a 32-year-old man.

\section{CASE REPORT}

A 32-year-old male attended the outpatient department of otorhinolaryngology with the complaint of nasal obstruction in the left nostril for two months. He had no history of nasal bleeding, hyposmia, facial pain and allergies. Anterior and posterior rhinoscopy showed smooth surface mass obstructing the left nasal cavity. He had no evidence of neck node enlargement and other clinical examinations within normal limits. Diagnostic nasal endoscopy confirmed the mass arising from the posterior end of the left side of the nasal septum. A computed tomography (CT) scan with contrast of the nose and paranasal sinuses was done and showed a mass with nonhomogeneous enhancement at the posterior part of the nasal

\section{Corresponding Author:}

Prof. Santosh Kumar Swain, Professor, Department of Otorhinolaryngology, IMS and SUM Hospital, Siksha "O” Anusandhan University, K8, Kalinganagar, Bhubaneswar-751003, Odisha, India; Cell: +91-9556524887; Email: santoshswain@soa.ac.in

ISSN: $2231-2196$ (Print)

Received: 09.02 .2021
ISSN: 0975-5241 (Online)

$$
\text { Revised: } 15.03 .2021
$$

Accepted: 02.04 .2021

Published: 26.05 .2021 
septum (Fig. 1). The mass revealed 1.5 X $4.8 \mathrm{~cm}$ soft tissue mass and showed no evidence of bone erosion. A small piece of tissue was taken from the nasal septum mass under local anaesthesia for histopathological examination. Cut a section of the mass showed smooth, pale white glistening neoplasm with streaks of haemorrhage. The histopathological examination confirmed benign schwannoma. The histopathological report showed spindle-shaped cells arranged in bundles, sometimes mimicking the palisade (Fig.2). Immunohistochemical study showed positive for S-100 protein and vimentin whereas negative for desmin and smooth muscle actin. The diagnosis of benign schwannoma was confirmed. The patient underwent endoscopic excision of the tumour from the nasal septum. Follow up of the patient after 3 months of surgery showed healed nasal cavity with clinical evidence of recurrence.

\section{DISCUSSION}

Schwannomas are infrequent and benign neurogenic neoplasm which originates from the nerve sheath of the myelinated nerves and found at any peripheral, cranial or autonomic nerves which contain Schwann cells. Schwannoma was first described by Verocay in 1910, who coined this benign neurogenic tumour as neuroma. ${ }^{4}$ However, the term schwannoma was proposed by Del Rio-Hortega in 1942 to indicate this tumour arising from the Schwann cells. ${ }^{5}$ The nasal septal schwannoma was first documented by Bogdasarian and Stout in 1943. ${ }^{6}$ Other terminologies used for this tumour were neurinoma, neuroma, neurilemmoma, neurilemmoma, perineural fibroblastoma, peripheral glioma and schwannoglioma, but are presently considered as inaccurate and obsolete which indicate lesions containing all the elements of a nerve fibre. ${ }^{7}$ This tumour may arise at any age but the peak incidence is between the third and sixth decades without any sex predilection. ${ }^{3}$ This tumour may occur alone or associated with the genetically inherited disease: neurofibromatosis type $1(\mathrm{NF} 1)$ or type 2 (NF2) and schwannomatosis.NF2 gene acts as a tumour suppressor and regulates Schwann cells. ${ }^{8}$ The most common origin of the head and neck schwannomas is the VIIIth cranial nerve. These tumours are extremely uncommon in the nasal cavity. Less than $4 \%$ of the schwannoma of the head and neck region are found in the sinonasal tract. ${ }^{8}$ These neoplasms include schwannoma (neurilemmoma or neurinomas), neurofibromas and traumatic neuroma. Schwannoma usually affects the posterior part of the nasal septum and is likely to originate from the nasopalatine branch of the trigeminal nerve.

Most of the head and neck schwannomas present as slowly enlarging, solitary, non-tender and encapsulated mass. Schwannomas are benign and encapsulated tumour which arises from the spinal roots, cervical nerves, sympathetic nerves, vagus, peroneal and ulnar nerves. The clinical presentations of the patient with nasal septal schwannoma de- pend on the site of the origin and the extent of the tumour. The common symptoms include unilateral progressive nasal obstruction, intermittent epistaxis, local pain, mucopurulent nasal discharge, anosmia, facial swelling, proptosis and pain in the sinonasal area. ${ }^{9}$ The symptoms are usually determined by the location and size of the tumour.

The differential diagnoses of the schwannoma in the head and neck region are soft tissue tumours, malignancy and salivary gland tumours. ${ }^{10}$ The differential diagnosis of the nasal septal schwannoma includes nasal polyps, concha bullosa, inverted papilloma, retention cysts, antrochoanal polyp and chronic rhinosinusitis. ${ }^{10}$ Other less common differential diagnosis includes fibrous dysplasia, mucocele, pleomorphic adenoma, myxoma, lymphoma, ameloblastoma and squamous cell carcinoma. ${ }^{11}$

CT scan picture is not specific for the diagnosis of schwannoma. However, a CT scan is helpful to identify the site and extent of the tumour.MRI is useful over CT scan for differentiating the tumours from the inflammatory lesions and normal tissues. In addition to this, MRI is also helpful for assessing the intracranial invasion of the tumor. ${ }^{12} \mathrm{As}$ the imaging is very non-specific for schwannoma, the diagnosis of the nasal septal schwannoma depends on the histopathological report. The definite diagnosis of the schwannoma is done by histopathological examination. The use of the hematoxylin and eosin staining of the schwannomas will show Antoni A or B cell types structures. Insinonasal schwannoma shows tight organizing palisading called Verocay bodies. Antoni B cell is typically characterized by loose, myxoid stroma running in an irregular pattern with spindle cells throughout. ${ }^{13}$ Macroscopic picture of the schwannoma appear as well-circumscribed, encapsulated, cystic mass which is connected to the nerve tissue. Microscopically it is classified into two types: Antoni A and Antoni B patterns. Antoni A patterns consist of spindle cells organized as cellular areas with nuclear palisading. Antoni B pattern is characterized by disorganized $b$, loose myxoid stroma with few spindle cells. ${ }^{14}$

The treatment option for nasal septal schwannoma is surgical excision which can be done lateral rhinotomy or endoscopic endonasal surgery. ${ }^{9}$ The endoscopic endonasal technique with or without image guidance is the standard surgical approach for the removal of the tumour. The advantages of endoscopic endonasal surgery are avoidance of the external incision, excellent visualization, minimal trauma to the surrounding tissues, shorter hospital stays lower morbidities in comparison to the external approaches. This clinical entity is typically curative and postoperative recurrence is extremely rare. ${ }^{15}$

\section{CONCLUSION}

The schwannoma at the nasal septum is extremely rare in routine clinical practice. It can be considered as a differential 
diagnosis in benign lesions of the sinonasal tract. The final diagnosis is confirmed after histopathological examination and immunohistochemical analysis. The treatment of choice is complete excision of this tumour and it has an excellent prognosis. These tumours usually do not show recurrence after complete surgical excision. Currently, the trans-nasal endoscopic approach is considered the most effective approach for nasal septal schwannoma.

\section{Acknowledgement: None}

\section{Conflict of interest: Nil}

Funding: No funding sources were granted or used specifically for this work.

Author Contribution:SKS: Concept, data collection and data analysis; DK: Data collection and drafting.

\section{REFERENCES}

1. Swain SK, Debta P, Sahoo S, Samal S, SahuMC, Mohanty JN.A rare site for oral cavity schwannoma: A Case Report.Ind J Pub Health Res Devp. 2019;10(11):1022-1025.

2. Yang TL, HsuMC, LiuCM.Nasalschwannoma: A case report and clinicopathologic analysis.Rhin. 2001;39(3):169-172.

3. Swain SK, Samal S.An unusual location of schwannoma in a 4-year-old child.Med J Dr.DY Patil Vidyap. 2020;13(4):406409.

4. Lollar WK, Pollak N, Liess DB, Miick R, Zitsch R. Schwannoma of the hard palate. Am J Otolaryn. 2010; 31(2):139-140.

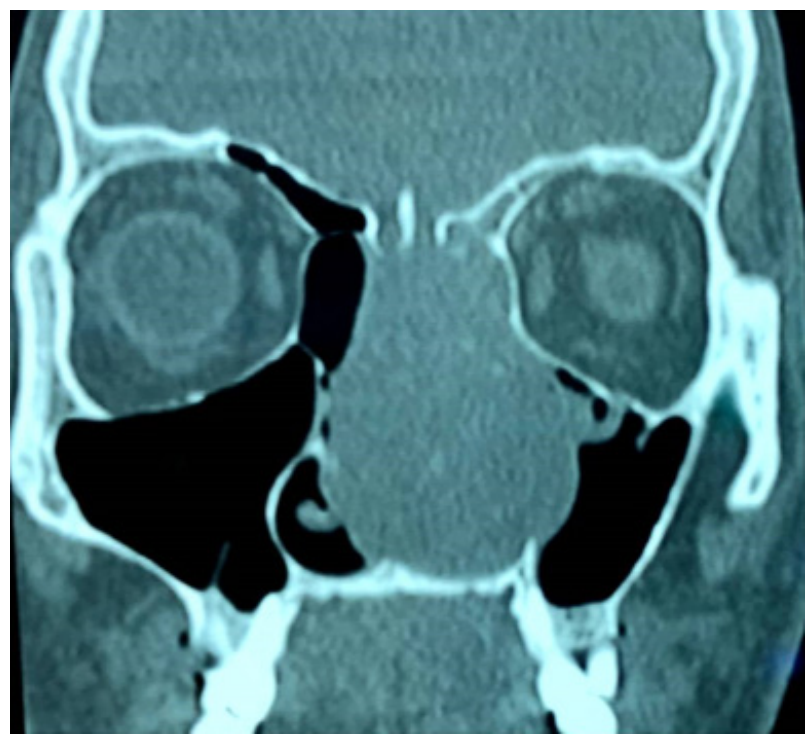

Figure 1: CT scan of the nose and paranasal sinuses showing the tumor arising the nasal septum.
5. Del Rio-Hortega P. Charactereseinterpretation de lascelulasespecificas de los neurinomas. Arch Soc Argent Anat.1942; 4(1):103-105.

6. Bogdasarian RM, Stout AP. Neurilemmoma of the nasalseptum. Arch Otolary.1943;38(1):62-64.

7. Pauna HF,CarvalhoGM,GuimaraesAC,MaunsellRC,SakanoE Schwannoma of the nasal septum:evaluationof unilateral nasal mass.Braz J Otorhin. 2013;79(3):403.

8. Ferner RE. Neurofibromatosis 1 and neurofibromatosis 2: a twenty firstcentury perspective, Lancet Neurol. 2007(6): 340351.

9. Mey KH,BuchwaldS,DaugaardS,PrauseJU.Sinonasal schwannoma-a clinicopathological analysis of five rare cases.Rhinol. 2006;44(1):46-52.

10. Martins DM, Jesus LA, Fernandes SPK, Bussadori KS, Taghloubi AS, Martins M. Intraoral schwannoma: case report and literature review. Ind J Dent Res. 2009; 20(1):121-125.

11. HabesogluTE,Habes ogluM, SurmeliM,Uresin T, Ege liE.Unilateralsinonasalsymptoms.J Craniofac Surg. 2010;21(6):2019-2022.

12. Swain SK, Agrawala R. Intraoral schwannoma in a seven-yearold boy- a case report.Pediatr Pols. 2020; 95(1):52-55.

13. Zhou P, Zeng F, Li J, Liu S. Only septal deviation? A tiny schwannoma in the nasal septum. Indian J Otolar Head Neck Surg. 2013; 65(1): 80-82

14. Fine SW, McClain SA.Immunohistochemical staining for Calretinin is useful for differentiating schwannomas from neurofibromas. Am J Clin Pathol. 2004;122(4):552-559.

15. Batra PS, Luong A, Kanowitz SJ, Sade B, Lee J, Lanza DC, et al.Outcomes of minimally invasive endoscopic resection of anterior skull base neoplasms.Laryngoscope. 2010;120(1):9-16.

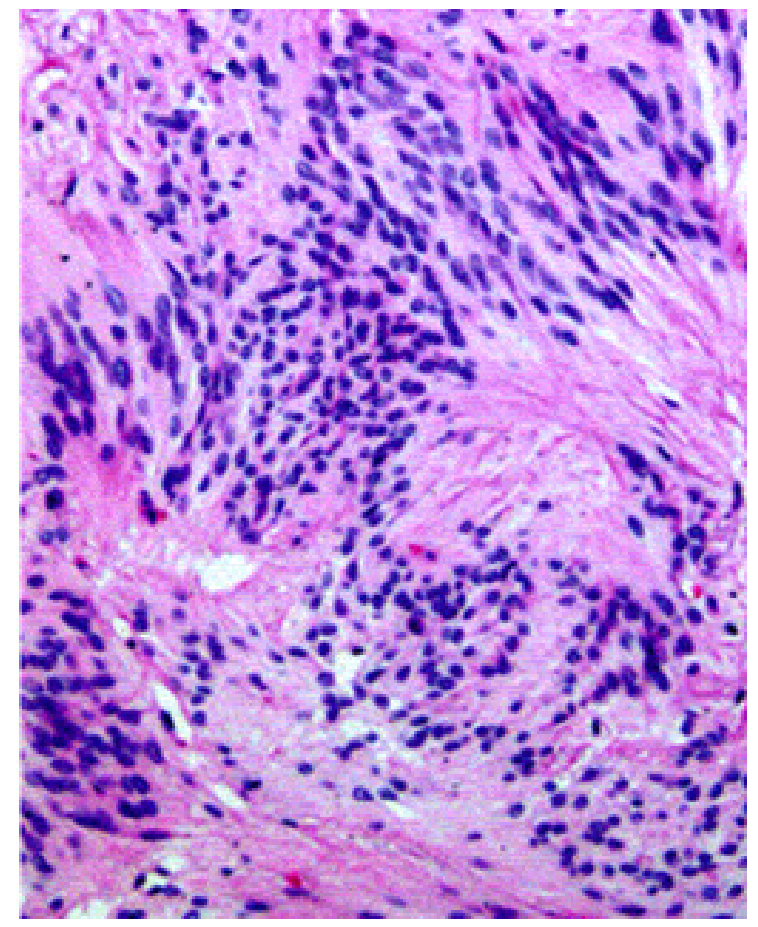

Figure 2: Histopathological picture showing the nasal septal schwannoma. 